\title{
HACIA UNA NUEVA FuSión CONCEPTUAL ENTRE ECONOMÍA Y EMPRESA: ECONOMIAA EMPRESARIAL
}

- Colectivo de profasares dal Programa Econornia Empresarial UAM

\section{Palabras Clave:}

Empresariado, Empresario, Empresa, Entorno, Relaciones, Sistema, Formal, Complejo, Dinámico, Campos de Acción, Objeto de Estudio, Perfil Profesional.

\section{RESUMEN}

\section{Introducción.}

Frente a los desafios que nos impone el mundo de hoy, el programa de Economia Empresarial, después de veinte años de experiencia y tomando en cuenta las tendencias actuales de los programas de Economia y de Administración, ha diseriado un curriculo que responde a una nueva delimitación de un campo del conocimiento, esta nueva delimitación es distinta de la de Economía y de la de Administración, con objeto de estudio
- Este trabojo fue realizado por el equipo de prufesores del Programa de Economía empresarlal durante va año de reuniones semanales en las cuales se discutieron 105 temay, los conceptos fundamentales y se tiegaron a los acuerdos aqui presentados. El recopilador y escritor de esta propuesta es el profesor José Jesús Naranjo G, del Departamenta de Clenclas Humanas:

$\therefore$ tales como la globalzactón, la necesidad de pensar lo regional y su inserción en 10 global, is socledad del conceimiento, el cambio vertiginosa de los modos de procucción $y$ de las relaciones sociales preducto det avance clentifico y tecnologico, la incertidumbre y las urgertes necesidades del hambre $y$ dei planeta,

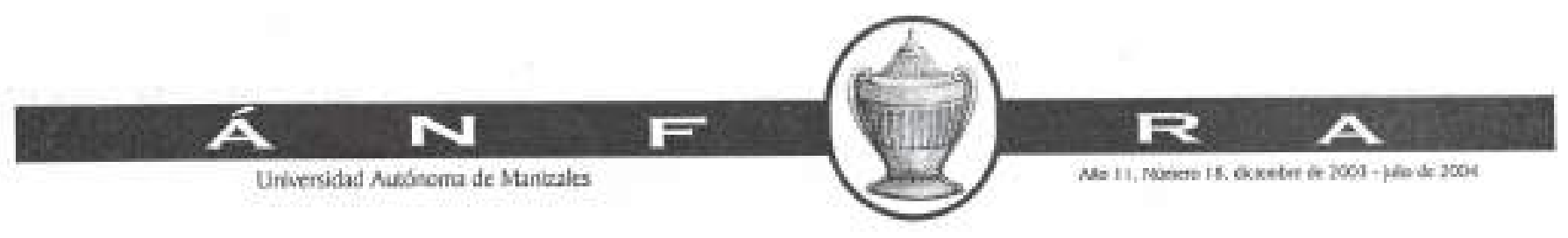


propio. El programa logra asi claridad en su quehacer académico, en el administrativo y en la formación de sus educandos.

Esta demarcación surge de un proceso de reflexión profunda y continua entre quienes conformamos los diversos grupos de estudio y las comunidades académicas de la Facultad, en reuniones semanales de mucha discusión y exposición de conocimientos desde las diversas posturas, producto de las teorias más aceptadas hoy en el mundo empresarial. De esta manera, delimitamos los campos propios de la Economía, la Administración, la Contaduria, el Derecho (en lo referente a la empresa y la economia), las finanzas, para liegar a la comprensión y delimitación del campo propio de la Economia Empresarial.

Este nombre el de Economia Empresarial lo usamos en defecto del de Estudios Empresariales, ya que al otorgar el título de Empresario, que seria el propio de esta carrera, se confundiria con su significado habitual; hombre que posee una o varias empresas; por tal motivo, el titulo que ofrece el programa es el de Economista Empresarial, pero entiéndase por ello "empresario".. Quede asi explicado, que Economia Empresarial no es una mezcla de economía y empresa o de economia y administración o de empresas y finanzas como aparece en otras escuelas que llevan el mismo nombre en diversos lugares del planeta, sino que responde a los estudios sobre el empresariado, a cómo y bajo qué teorias, habilidades y destrezas se debe formar al empresario para responder a los retos del mundo actual. De tal manera que, el Economista Empresarial, es un hombre o una mujer cuya profesión es ser empresario(a). Esto es, no un gerente ni un administrador ni un economista sino un creador y dinamizador de empresas.

\section{Demarcación del campa de conocimiento de Ia Economia Empresarial}

Si la Economia estudia las relaciones sociales en cuanto a los modos de producción y distribución de unos recursos siempre escasos y el campo de conocimiento de la Administración es la organización, la Economia Empresarial se enmarca dentro del campo del conocimiento que comprende las relaciones sistémicas, formales, dinámicas y complejas entre EMPRESA EMPRESARIO ENTORNO, tomando como objeto de estudio el EMPRESARLADO cuyo fin es el de formar los hombres y mujeres capaces de establecerse por cuenta propia que generen el progreso que requiere la región, el pais y el mundo globalizado de hoy.

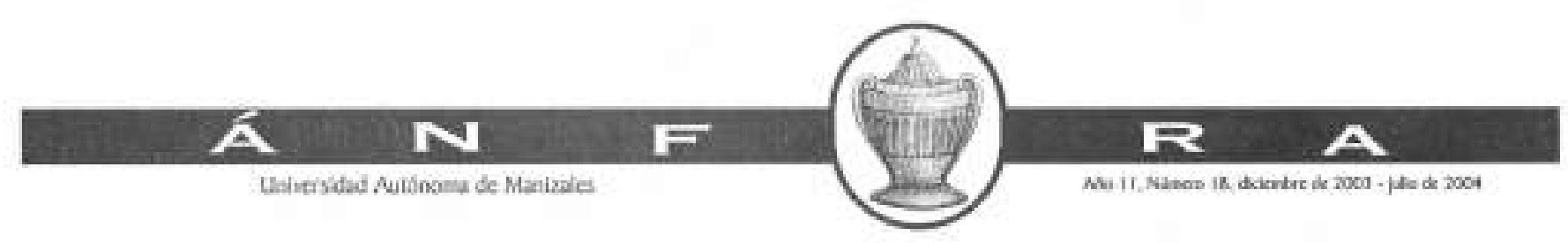




\section{Nueva profesión: serempresario.}

Es innegable que debido a las necesidades de hoy, sobre todo por la crisis general que atraviesa no sólo el pais sino el mundo, los programas de Administración y de Economia tienden a formar en sus educandos el espíritu empresarial con la esperanza que estos profesionales conformen empresas, generen emploo y den alternativas de cambio con el fin de subsanar dicha crisis. Pero también es cierto que con los fundamentos de economía solamente o con los de administración, no se forman empresarios, asi hagan énfasis en el desarrollo de la capacidad empresarial o en despertar y afianzar el espiritu empresarial. Se necesitan fundamentos teóricos y prácticos en empresariado para formar jóvenes capaces de desempeñarse en esta nueva profesión: ser empresario. Una profesión que sólo ejercen algunas personas de manera intuitiva, arriesgada y generalmente por herencia. $Y$ es precisamente aqui, donde la Universidad debe estar en capacidad de tomar esos saberes no académicos ni cientificos y estudiarlos, sistematizarlos y darles el rigor propio de la academia para ofrecer una educación de alta calidad a quien desee desenvolverse en este campo de acción.

Este es nuestro esfuerzo y nuestro empeño: crear un ambiente de aprendizaje propio para la formación de empresarios. No es sólo un éntasis, como lo sugieren algunas facultades de administración en el pais, sino el núcleo mismo de ia formación, de tal manera que el microcurriculo o lo que es lo mismo, las materias propias y los procedimientos y politicas del programa de Economia Empresarial con sus intenciones, finalidades, formas de comunicación y demás características del curriculo- está compuesto por: empresariado (historia, qué es, cómo es, para qué es; empresario: definición, clases, cualidades, caracteristicas), Historia empresarial (historia de empresas, de épocas de creación de empresas), Historia de empresarios, filosofia empresarial, Espiritu emprendedor I y II (que incluye creatividad, innovación, liderazgo, construcción de pensamiento, psicología social sobre todo por el proceso grupal y el trabajo en equipo), Gestión del Cambio, Plan de negocios, Toma de decisiones y Riesgo. Como complemento de la formación diseñamos el mesocurriculo, compuesto por los conceptos fundamentales $y$ pertinentes (minimos óptimos) de economia, administración, derecho, marketing, finanzas y matemáticas. Por último, contamos con el macrocurrículo que ofrece la formación humanista y en idiomas extranjeros.

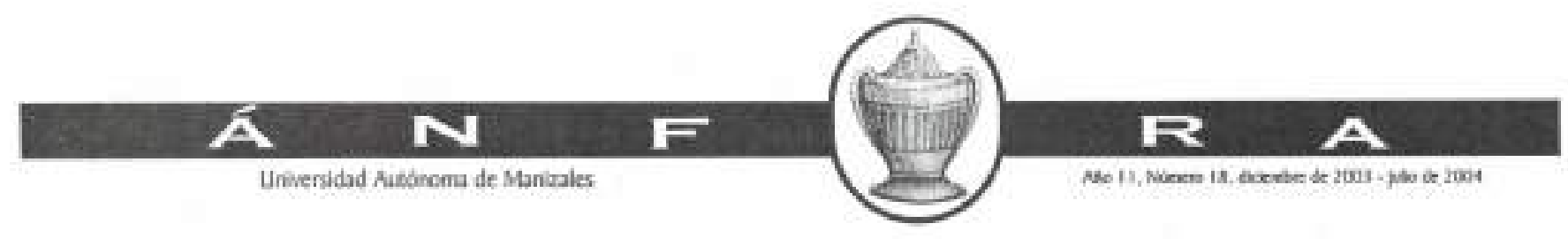




\section{Definición de Economía Empresarial.}

Eccnomia Empresarial es un programa de la Facultad de Ciencias Sociales y Empresariales de la Universidad Autónoma de Manizales que estudia e investiga cómo hacer posible la formación de empresarios que, al crear y trasformar empresas, responden a las necesidades de la región y del país para mejorar la calidad de vida de sus habitantes.

Existen en el mercado educativo, según información obtenida en la internet, otras escuelas de Economía Empresarial como en la Universidad Anáhuac del Sur (México), en la Universidad de Zaragoza (España) y en el MIT en Estados Unidos y su filial UTDT del Brasil, que ofrecen este programa, pero como una asociación entre economía y empresa, entre empresa y entorno, y entre administración y economia. Mientras que nuestro programa tiene claridad en cuanto al propósito de formación que consiste en formar empresarios, basados en el objeto de estudio que denominamos empresariado y que se enmarca dentro de la región de conocimiento comprendido entre las relaciones sistémicas, formales, dinámicas y complejas de la empresa, el empresario y el entorno.

\section{Misión.}

El programa de Economía Empresarial tiene como misión estudiar, crear y recrear un ambiente de aprendizaje en el que se propicie el desarrolio teórico práctico de su objeto de estudio para contribuir a la formación de empresarios éticos con pensamiento crítico e innovador, lideres creadores o dinamizadores de empresas.

Entiéndase aqui "dinamizadores de empresas" como la capacidad del egresado para ser intraempresario, uno de los posibles campos de acción del futuro profesional que consiste en tener los conocimientos y las habilidades necesarias para intervenir en empresas ya constituidas pero que requieren de una nueva visión, de una profunda reestructuración basada en un nuevo direccionamiento estratégico para salir avantes en el medio. Son el ingenio y la creatividad los posibilitadores de este cometido.

\section{Visión.}

En el año 2007 el programa de Economía Empresarial de la Facultad de Ciencias Sociales y Empresariales de la Universidad Autónoma de Manizales, será una unidad académico-administrativa sólida que brinda una excelente educación teórico-práctica y humanista para la formación de empresarios comprometidos con la región, pensada ésta desde los retos de la globalización. Asi, este programa, se convertirá, al ritmo de la Universidad, en pionero del desarrollo regional.

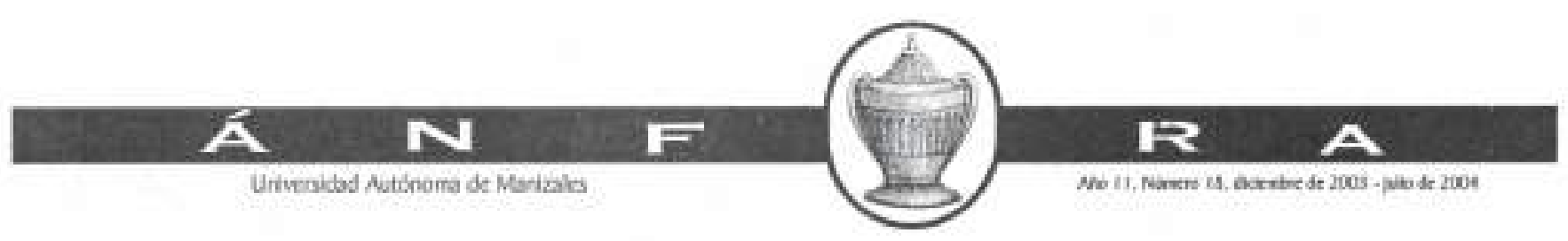




\section{Objetlvogeneral del programa}

Contribuir a la formación de empresarios con pensamiento critico e innovador, líderes en la creación y... O... transformación de empresas, mediante una fundamentación teórica práctica - humanista de excelencia, para generar profesionales capaces de trabajar por el crecimiento socioeconómico sostenible de la región y del pais.

\section{Ohjetivas especificos.}

2. Desarrollar y... o... despertar el espíritu empresarial en sus educandos, mediante un estudio concienzudo de las particularidades del empresario y las prácticas requeridas para dicho resultado, con el fin de garantizar los conocimientos, las actitudes, las capacidades y las habilidades necesarias para la creación $y . . .0, .$. transformación de empresas.

Q. Potenciar el liderazgo en los alumnos, por medio de un ambiente de aprendizaje adecuado para hacer germinar una de las características propias del empresario.

\& Generar un pensamiento crítico e innovador, a través de la estrategia pedagógica de los cuatro saberes, pilar fundamental de la educación impartida en el programa, para reunir las condiciones necesarias de un excelente empresario, consultor, intraempresario y... o.... investigador.

\& Formar empresarios éticos, humanistas y solidarios, mediante las cátedras que ofrece el departamento de Ciencias Humanas, para lograr un profesional idóneo y de alta calidad humana.

2. Educar en la libertad, en la autonomia y en la responsabilidad, mediante ejercicios teórico prácticos, para la toma de decisiones, factor éste indispensable en el empresario ético que tiene obvia conciencia de su responsabilidad social al tomar decisiones que pueden afectar a una o muchas personas, lo mismo que a la región y al país..

\&. Preparar a nuestro profesional para afrontar el riesgo propio de un entorno cambiante, con el fin de incentivar la toma de decisiones ágiles, eficientes, eficaces y seguras.

\section{Objetode estudio.}

El Objeto de Estudio de la Economia Empresarial es el Empresariado que como núcleo, reúne las relaciones sistémicas, formales, dinámiças y

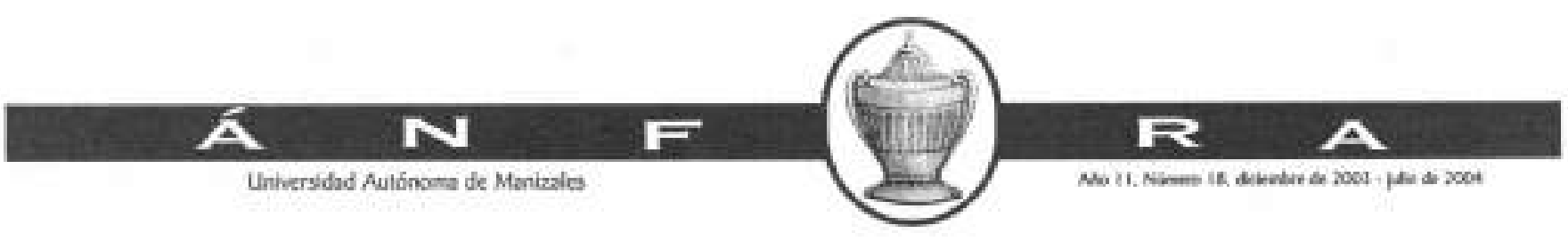


complejas entre EMPRESA EMPRESARTO ENTORNO y es condición sine qua non se es empresario.

El Empresariado como objeto de estudio puede analizarse desde los componentes cognitivo, actitudinal y organizacional. El componente cognitivo abarca los conocimientos fundamentales sobre el espiritu emprendedor, la capacidad de interpretación, de leer el entorno, de localizar, reconocer, seleccionar, procesar y utilizar información, la capacidad para tomar decisiones con base en la información, la capacidad de investigación, de aprender a aprender, de reconocer las propuestas mágicas de certidumbre para desmitificarlas y resignificarlas, porque el mundo empresarial fluye más en la incertidumbre y el riesgo que en la seguridad y la certeza. El segundo componente, el actitudinal comprende la creatividad, la innovación, el riesgo, la responsabilidad social, la sensibilidad humana, social y ambiental. Finaimente, el componente organizacional tiene que ver con la administración, la economia, la eficiencia, la eficacia, el trabajo en equipo, la coordinación de recursos y talentos.'

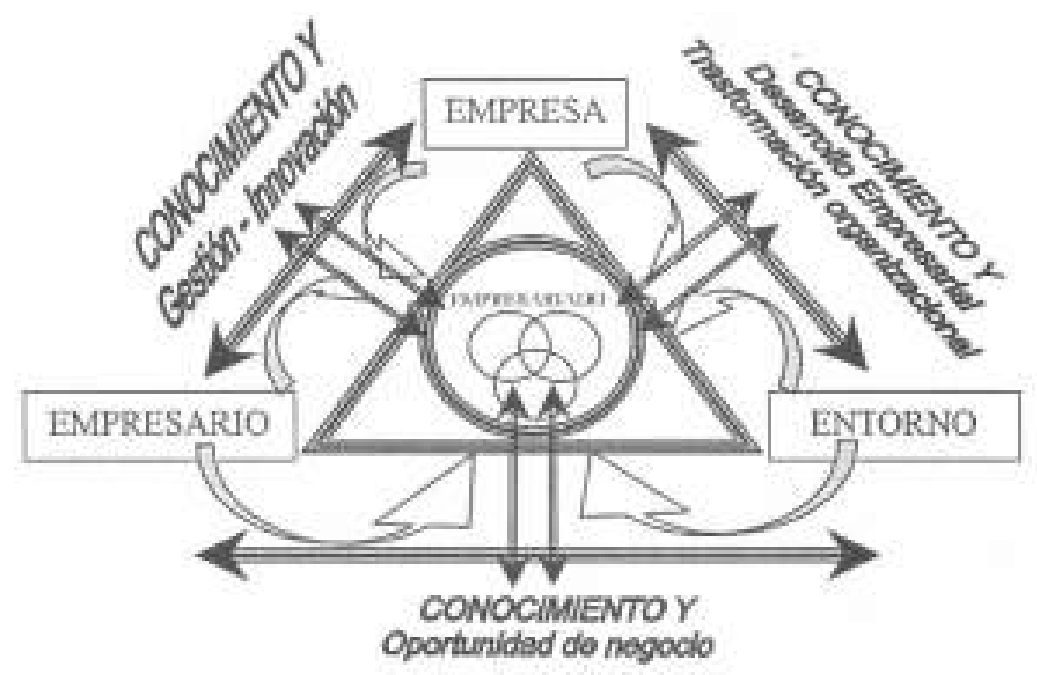

\section{Campos de acción del Economista Empresarial.}

Por acuerdo con los profesores del programa y contextualizando el concepto de paradigma de Khun, como ese conjunto de compromisos compartidos por la comunidad académica, se han definido como campos de acción de la Economia Empresarial:
'Los tres circulos interceptados represertan los componertes cognitivo (racional), actitudinal (emotivo) $y$ organizadional (praxlológlca) de) empresariado. La división solo se hace para fines explicativos pero que en el ser humano se presentan a: unisono, pues su base de funcioramiento es el cerebro yestees halistico.

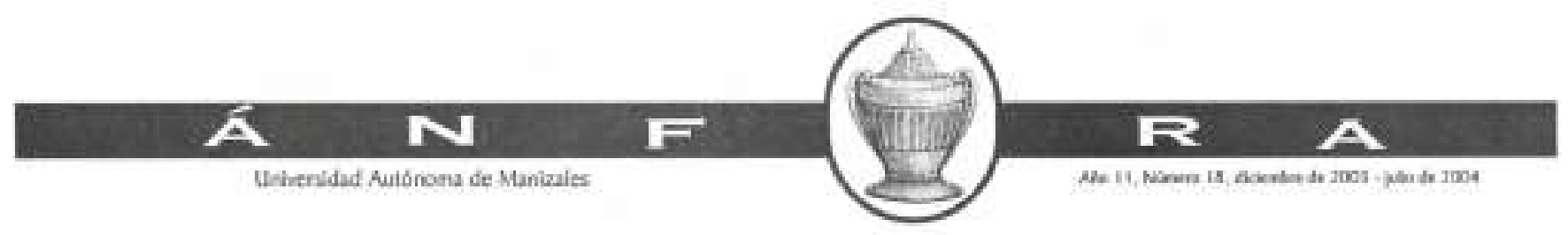




\section{La Creación de Empresas.}

Es, a la vez, el centro de la formación en el programa. Capacitar a los educandos para que tengan no sólo los conocimientos necesarios sino también las habilidades, destrezas y actitudes que les permita tomar el riesgo de invertir en la creación de empresas e impactar positivamente la región y por ende el pais, es decir, el entorno.

\section{Elintraempresariado.}

Entendiendo por éste, la posibilidad de penetrar en las empresas para trasformarlas, en palabras nuestras, generar empresa dentro de ia empresa. Tener la capacidad de innovar y renovar para sacar adelante empresas estancadas o en franco camino de recesión.

\section{LaAsesoria.}

El profesional egresado de nuestro programa cuenta con una rigurosa formación cientifica que unida a la habilidad para ubicar, seleccionar y manejar la información, al pensamiento critico e innovador, a la capacidad para la resolución de problemas, al entrenamiento en la planeación estratégica $y$ al manejo de diversos métodos de intervención, puede convertirse en experto que ofrece asesoría a las empresas.

\section{La Investigación.}

La Universidad Autónoma de Manizales, consciente de su papel educador, investigador $y$ de proyección ha tomado en serio la necesidad de crear $y$ fomentar los grupos de estudio, los grupos académicos y los de investigación en los que participan los alumnos. El Departamento de Ciencias Humanas ha iniciado desde este año (2.002) una experiencia mediante la cual todas sus cátedras se convierten en programas de investigación y asi familiariza a todos los educandos de la Universidad con el lenguaje y los métodos propios de la investigación, de tal forma que los egresados de Economia Empresarial no son ajenos a las faenas propias de la investigación. De igual manera, la Facultad de Ciencias Sociales y Empresariales se ha preocupado por crear, desarrollar y motivar una cultura del asombro y la curiosidad, bases éstas de lo5 procesos investigativos. Cuenta la Facultad, además, con la linea de investigación en empresariado con varios grupos de estudio, entre elios el de finanzas, el de marketing, etc., lo que afianza el perfil investigador del egresado.

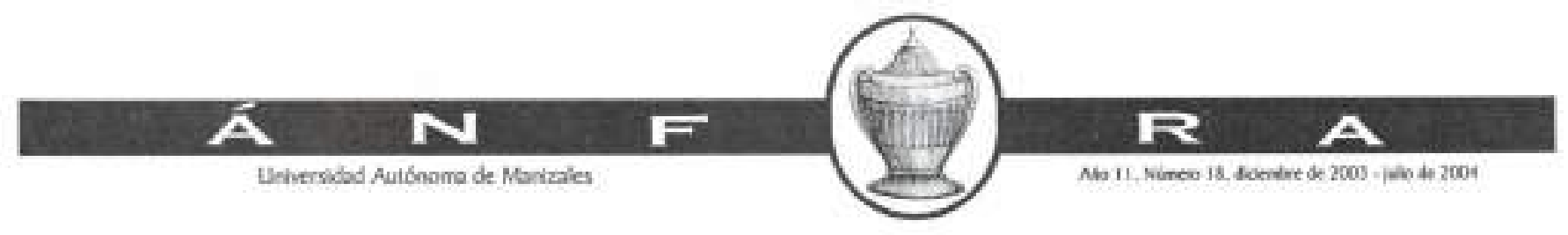




\section{Perfil profesional.}

El Economista Empresarial de la Universidad Autónoma de Manizales es un empresario ético, con mente estratégica, pensamiento crítico e innovador. Un lider con conocimientos sólidos sobre su profesión que asume con excelencia los retos que le impone el entorno.

Si tenemos en cuenta las definiciones sobre empresario de I. A. Schumpeter, N.J. Devenport, B.S. Klestread, Dale, A. H. Cole, La Confederación de Empresarios de Galicia, Winston Churchill, Juan Torras, Jean Baptiste Say, Richard T, Ellis y Ralph h. Hess, David McClelland, Kira Draheim, William Baumol, Orvis Collins y David G. Moore, Albert Shapero, Harvey Leibenstein, Kart Vesper, Gordon Baty, Robert Ronstadt, Jeffrey A. Timmons y del Servicio Nacional de Aprendizaje (SENA); además si tenemos en cuenta los escritos de Lipman, Rosell y otros, Enrique Sánchez, Rodrigo Varela, Drucker, Ohmae, Manfred Max-Neff, Reichheld y otros estudiasos del Empresariado, la Economia y la Administración, se puede diferenciar nítidamente cierto perfil para el empresario que forma el Programa de Economia Empresarial y se puede, también, reunir las mejores ideas sobre las condiciones, teorias, habilidades y destrezas que identifiquen con propiedad $y$ le den sello a nuestro egresado.

Veamos qué pensamientos tienen estos autores mencionados y qué pautas nos dan para pensar el perfil del Economista Empresarial:

Q J.A. Schumpeter: "Se enfoca a la creatividad tecnológica innovación y no al suministro de capital o en la mera arrogación de responsabilidad para hacer decisiones, to que significa que las decisiones deben tener un elemento de novedad, de diferencia para que la persona que las toma merezca el apelativo de empresario" (en Lipman, 1996). Podemos inferir aqui un énfasis en lo creativo e innovador, más que en el apego a teorias que en este terreno cambian con mayor prontitud que en otras ramas del saber. Por esto, la necesidad de aprender a aprender, de aprender a desaprender y de aprender a reaprender; de saber ubicar, manejar y contextualizar la información con presteza.

Q N.J. Devenport: "El empresario es el Gerente independiente y desempleado; es el que lleva los riesgos y denuncia las ganancias de la empresa" (en Lipman, 1996). De esta idea se rescatan la necesidad de prepararse para el 'riesgo', el carácter de 'independiente' y el rasgo de 'desempleado', más que el de 'gerente' y 'denunciador de ganancias'.. Aunque la gerencia, la administración y la economia no resultan ajenos al empresariado, el programa de Economia Empresarial al hacer claridad sobre su objeto de estudio, hace énfasis en los rasgos y caracteristicas

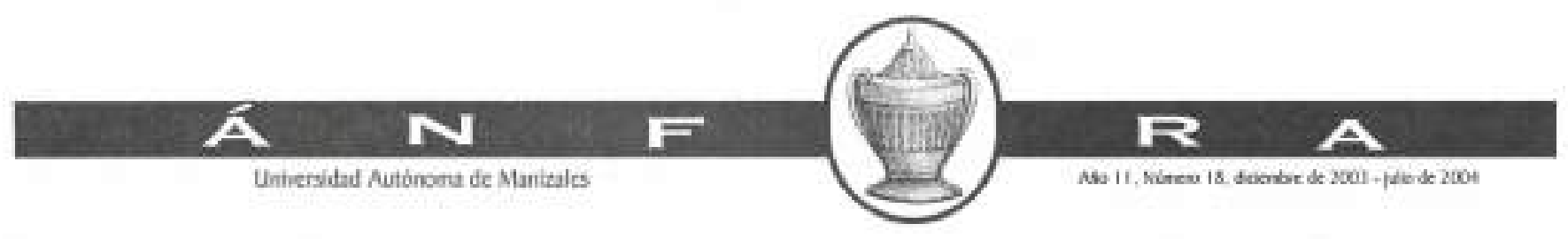


más sobresalientes de un empresario y éste debe tener un perfil que apunte más al desarrollo de la capacidad empresarial que al conocimiento dogmático de doctrinas económicas, sobre organizaciones o de gerencia sin descartar la necesidad de tener un dominio suficiente sobre el conocimiento disponible hoy para la creación y marcha de las empresas.

Q B.S. Kiesrtead: "La función del empresario es la de tomar decisiones que gobiernen la politica de la firma sobre la fuerza de sus expectaciones acerca del futuro".. (en Lipman, 1996). De esta afirmación podemos resaltar 'la toma de decisiones' como fundamental en la formación del futuro empresario. Las preguntas que surgen, entonces, son sobre ¿cómo es posible capacitar en la 'toma de decisiones'? ¿qué conocimientos se requieren? ¿es una competencia? ¿cómo se desarrolla?

\& Dale: "Persona o grupo de personas que toman las decisiones en los negocios; y el empresariado la creación de las decisiones en la esfera de los negocios". (en Lipman 1996). Vuelve a aparecer aqui la idea de 'toma de decisiones', reiterativa también en el pensamiento de muchos estudiosos del empresariado y de las ciencias económicas, por eso vale la pena tener en cuenta su grado de importancia en la formación de los futuros profesionales. Otra idea que se encuentra en la definición de Dale, es la de 'creación de las decisiones' en la que está comprometida la creatividad de aquél que toma las decisiones.

2. A. H. Cole: "Aquellos que hacen, ejecutan y son responsables de las decisiones estratégicas de una empresa que persigue la ganancia". (en Lipman, 1996). De nuevo las 'decisiones' pero esta vez 'estratégicas' lo que implica una mente estratégica que las produzca y por tanto una formación para dicho fin.

Q. Confederación de Empresarios de Galicia (CEG): " ... una definición podría venir dada por la idea de empresario como la persona capaz de identificar una necesidad, de reunir los recursos necesarios y de emprender una acción para satisfacer una neccesidad". (CEG., 1996). La clave del empresario, según esta definición, está en la capacidad de 'identificar', de 'emprender' y de 'reunir' no sólo recursos sino talentos. Saber reunir talentos implica saber trabajar en equipo, otra pauta necesaria para la formación de empresarios.

Q Winston Churchill: "Muchos consideran al empresario como el lobo hambriento que hay que abatir, otros piensan que es una vaca a la que se puede ordeñar indefinidamente. Pocos son los que le consideran como el cabalio que tira del carro de la economia" (Churchill, 1950). De este famoso personaje, extraemos la idea del empresario como 'el caballo que tira del carro de la economia', es quien jalona los procesos económicos

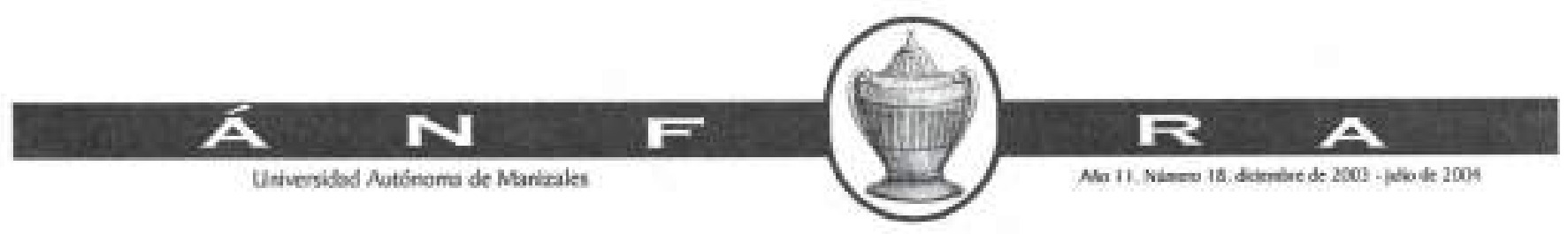


mediante las empresas que genera, mas no porque sea economista sino empresario. De ahi que, no debemos confundir al Economista Empresarial con su colega el Economista.

Q. Juan Torras: "...toda persona que toma decisiones en un mundo incierto y pone en fuego una parte de su capital humano, financiero o fisico que le pertenece o pide prestado con el objetivo de un resultado futuro favorable, entendido éste como la diferencia entre el valor actual de los desembolsos realizados y el valor actual descontado a la tasa de interés bruto de los ingresos futuros" Rescatable de esta definición la 'toma de decisiones' pero 'en un mundo incierto', se desprende, entonces, la necesidad de educar para la incertidumbre. El resto del contenido sólo muestra un economicismo a ultranza. Encontramos luego: "El empresario real es un 'especulador' un hombre capaz de utilizar su opinión acerca de la futura estructura del mercado para iniciar actividades empresariales capaces de generar beneficios" (Rosell y otros, 1985). Impresiona de esta cita el término 'especulador' que podria cambiarse por 'intuitivo', 'suspicaz', 'sagaz' y muchos otros términos ajenos a la academia pero que tienen que ver mucho con el espiritu empresarial y a los que el programa de Economia Empresarial de la Universidad Autónoma de Manizales les da la mayorimportancia.

2. Enrique Sánchez: "Empresario es la persona que arriesga su trabajo, capital o crédito para suministrar a la colectividad un bien o servicio deseado, transformando los factores productivos. En razón a la incertidumbre del mercado, si el servicio prestado es valorado por el consumidor a un precio mayor a su costo, obtendrá ganancia, en caso contrario, experimentará pérdida". (Sánchez, 1991). Es importante ver aqui la responsabilidad social del empresario, su función, o mejor su misión, es netamente social: satisfacer necesidades y 'deseos' a la colectividad. De aqui, la necesidad de una formación altamente humanista para preparar un profesional capaz de comprender lo humano y lo social. Es de anotar que aparecen nuevamente los términos 'riesgo' e 'incertidumbre'.

Q Rodrigo Varela: "El empresario es la persona o conjunto de personas que es capaz de percibir una oportunidad de producción o de servicio, y ante ella formula libre e independientemente una decisión de consecución y asignación de los recursos naturales, financieros, tecnológicos y humanos necesarios para poder poner en marcha el negocio, que además de crear valor incremental para la economia, genera trabajo para él y muchas veces para otros. En este proceso de liderazgo creativo, el empresario invierte dinero, tiempo y conocimientos y participa activamente en el montaje y operación del negocio, arriesgando sus recursos y su prestigio

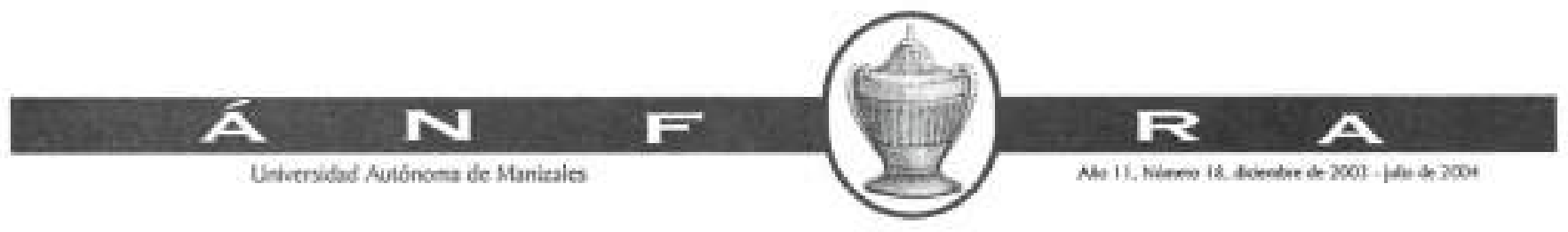


personal, pero buscando recompensas monetarias y personales. (Varela, 1991). Apreciamos de esta definición la necesidad de 'libertad' e 'Independencia', esto es, necesidad de formar en la autonomia, en la responsabilidad $y$ en la libertad al futuro profesional. Otro factor indispensable en la educación del empresario es el de 'liderazgo creativo'.. Se requiere despertar ese espiritu de liderazgo y desarrollar la capacidad creativa y de innovación. Una tercera idea extraida de esta definición es la de la 'autoestima' pues el empresario arriesga su 'prestigio personai' y debe estar preparado para esos momentos inesperados. La psicologia es una rama del saber que aporta muchisimo a la formación del empresario, por la preparación personal y de equipo que puede ofrecer. Nótese que vuelve a aparecer el término 'riesgo'; y no sin razón, el colectivo de profesores que dio origen a esta organización de ideas, piensa en la necesidad de implementar el deporte de alto riesgo como una técnica que prepara adecuadamente para el riesgo a los futuros empresarios.

e. Jean Baptiste Say (1803). "El empresario es el agente que reúne y combina los medios de producción (recursos naturales, recursos humanos y recursos financieros) para construir un ente productivo y encuentra en el valor recibido de los productos la recuperación del capital que él emplea de los gastos en que incurre y de la utilidad que busca. Es el punto de apoyo (pivot) sobre el cual gira todo el negocio. Debe poseer el arte de la superintendencia y de la administración" (Varela, 1991). Este tipo de pensamiento, según nuestras pesquisas, es más de un administrador o gerente que de un empresario, pues al poseer el "arte de la superintendencia' tiene el arte de dirigir, gobernar y administrar una empresa y no de crearla; sin embargo, es necesario rescatar, para nuestras ideas, la de ser un 'agente que reúne y combina los medios', esto implica la 'capacidad para ser recursivo' que debe poseer nuestro egresado.

Q. Richard T, Ellis y Ralph H. Hess (1893): * Definen al empresario (capitán de industria) como la persona o conjunto de personas que toman para si la tarea y la responsabilidad de combinar los factores de producción en una organización de negocios y de mantener la organización en operación. El empresario organiza y opera una empresa para su utilidad personal; contribuye con su propia iniciativa, habillidad e ingenio a planear, organizar y administrar la empresa. Toma para si el chance de ganar o perder y se enfrenta a circunstancias imprevistas e incontrolables" (Varela, 1991). Se rescatan de aqui dos ideas: 1. la necesidad de preparar a los jóvenes empresarios en 'iniciativa, habilidad e ingenio' para planear, organizar y más que administrar la empresa sería para ponerfa a marchar. Hasta aqui su papel como empresario. Si decide continuar al frente de esa empresa, se convierte en administrador, para lo cual, el programa de

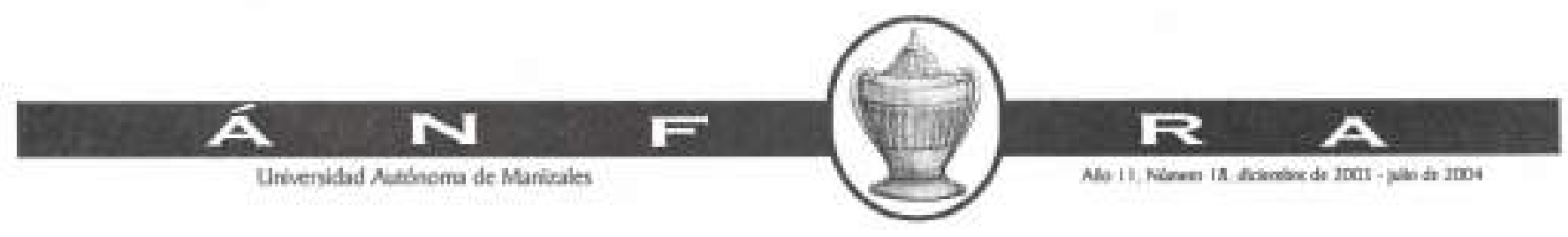


Economia Empresarial debe darle las bases, 2. el hecho que el empresario se enfrente a 'circunstancias imprevistas e incontrolables' nos hace pensar en la necesidad de preparar para lo inesperado, incontrolado e impredecible.

Q David McClelland (1961): "El empresario es alguien que ejerce control sobre los medios de producción y produce más de lo que consume con el fin de venderlo o intercambiarlo y lograr un beneficio para si" (Varela, 1991). De nuevo, esta es una mirada, además de economicista, netamente utilitarista y egoista. Hoy, el mundo exige una formación diferente que no sólo tenga en cuenta el 'beneficio para si' sino el impacto y beneficio social del trabajo del empresario. Ahora bien, esta definición sirve más para el negociante que para el empresario.

Q. Peter F. Drucker (1964): " "..indica que el trabajo del empresario es la maximización de oportunidades y que por lo tanto su función es determinar qué iniciativas deben ser realizadas (eficacia); y que se deben concentrar los recursos y los esfuerzos más en esas oportunidades empresariales que en los problemas gerenciales" (Varela, 1991). Drucker confirma las ideas que se han gestado al interior de la comunidad académica, al hacer diferencia de manera inconsciente, creemo5-entre empresario y gerente, aunque él llama a los empresarios, posiblemente de manera errónea, 'monomaniacos', y prefiere, claro está, porque su libro es sobre administración, a los gerentes; de todas formas afirma en su Mananging in a Time of Great Change, 1995: "Los gerentes [nosotros dirlamos, los empresarios] son sintetizadores que juntan recursos y tienen la habilidad de 'oler' las oportunidades. Hoy la perceptibilidad es más importante que el análisis." (p. 9) El carchete es nuestro. Pero, la pregunta fuerte es ¿cómo formar en la habilidad de 'oler' las oportunidades?

\&. Kira Draheim (1966): "El empresario es el iniclador activo de una nueva organización, juega un papel básico en empezar la compañia y en administrarla y generalmente tiene una participación en la misma*. (Varela, 1991). Esta afirmación confirma nuestros intereses y propósitos: formar iniciadores o creadores de empresas y de paso, brindar los conocimientos mínimos básicos para que puedan, dado el caso, administrarlas.

\& William Baumol (1968): "...considera que el trabajo del empresario es identificar nuevas ideas y ponerlas en marcha. Es la persona que lidera y es fuente de inspiración en el desarrolio de una nueva empresa". (Varela, 1991) Varias ideas rescatamos de este pensador. Una sería la capacidad de identificar nuevas ideas; otra, la necesidad del liderazgo; y una tercera, la inspiración. Ratificamos aquí la combinación entre arte y ciencia, entre

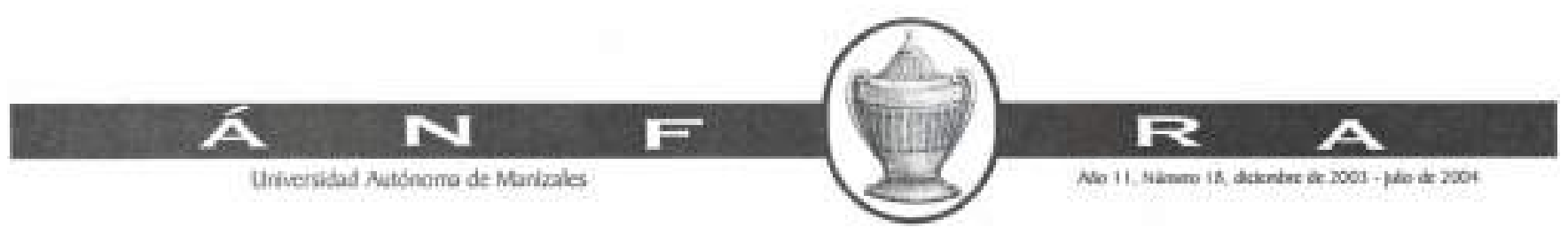


lo no racional y lo racional que se requieren en la formación de empresarios.

e. Orvis Collins y David G. Moore (1970): "El empresario independiente es el hombre que ha creado de la nada una organización en funcionamiento".. (Varela, 1991). Bueno, esta concepción si es como para magos, los únicos capaces de sacar de la nada un 'conejo'.. Una pregunta: ¿cómo se crea una organización en funcionamiento? Al menos una organización surge de una idea o de la capacidad de 'oler' una oportunidad de negocio y primero se planea, se estudia el contexto y las cifras, y luego se pone en funcionamiento. La creatividad nada tiene que ver con la magia. Aquélla exige mucho de transpiración y un poco de inspiración, mientras que ésta es truculenta.

\&. John Hornaday y John Aboud (1971): “...definen al empresario exitoso como el hombre o la mujer que emplezan un negocio donde antes no existia y lo desarrollan". (Varela, 1991). Preparar para la iniciación de empresas, ese es nuestro reto.

Q Albert Shapero (1975): "El empresario tiene como atributos: a) Toma de iniciativa, b) Organización o reorganización de los mecanismos sociales y económicos para convertir los recursos y situaciones en hechos prácticos, c) Acepta el riesgo de éxito o fracaso y d) Uno de sus recursos básicos es él mismo" (Varela, 1991). Aunque no define al empresario, nos da ideas valiosas sobre las 'atributos del empresario' que implican las relaciones entorno, empresa y empresario.

2. Harvey Leibenstein (1978): "...describe al empresario innovador como aquel que realiza las actividades necesarlas para crear una empresa, donde los mercados no están establecidos o definidos y en la cual la función de producción no está completamente conocida. Es un 'gapfilier', un 'input completer'". (Varela, 1991). Apostarle a lo desconocido, ver lo que no está, clarividencia o predicción, la una intuitiva, la otra cientifica son capacidades propias del empresario.

2. Kart Vesper (1980): Divide la definición de empresario según profesiones, asi:

36 "Para un economista es alguien que reúne recursos, mano de obra, materiales y otros activos para generar unos productos de mayor valor mediante cambios, innovaciones y nuevos ordenamientos"

8. "Para un empresario es un competidor agresivo y una amenaza, mientras que otro empresario lo ve como un aliado, un cliente, un proveedor y un bien prospecto de inversión"

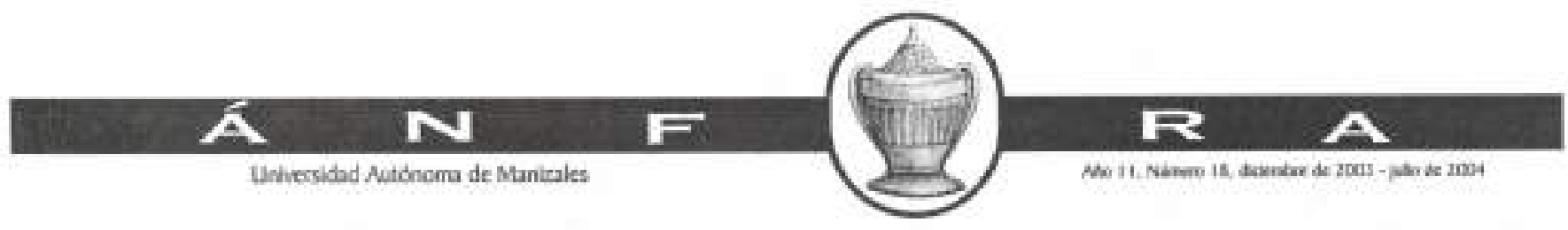


8 "Para algunos politicos el empresario es un rebelde, descarriado y muy dificil de controlar, pero otros lo ven como una persona que logra que las cosas se hagan"

8 Para un filósofo capitalista es una persona que crea riqueza para él y para otros, que encuentra las mejores formas de utilizar recursos y reducir desperdicios, alguien que produce trabajos que otros están felices de lograr" (Varela, 1991). El hecho que se piense al empresario desde otras profesiones, marca la diferencia entre éste y los demás profesionales. De aqui la posibilidad de crear y recrear unos saberes populares en saberes académicos y ese es precisamente el ejercicio que estamos realizando en la U.A.M.

2. Gordon Baty (1981): "...define la función empresarial como la planeación, el montaje, el desarrolio y la motivación y el éxito de la empresa" (Varela, 1991). Antes de la planeación se necesita la 'ideación' de la futura empresa to que implica, nuevamente, el componente creativo e innovador.

k. Robert Ronstadt (1984): "...define el proceso empresarial como el proceso dinámico de crear riqueza incremental. La riqueza es creada por individuos que asumen los principales riesgos en términos de capital, tiempo y compromiso personal para proveer valor incremental a sus productos o servicios. Dicho producto o servicio, puede o no ser nuevo o único pero el valor debe ser de alguna forma infundido por el empresario, consiguiendo y asignando los recursos y las habilidades necesarios. (Varela, 1991), En esta definición encontramos el componente dinámico que implica movimiento y éste implica cambio. Entonces, se debe tener en cuenta la educación para ese cambio, para interpretar e interrogar continuamente la realidad que presenta cada dia y de aquil la necesidad de saber leer el entorno de manera crítica para ver qué oportunidades se presentan.

Q. Jeffrey A. Timmons (1985): " crear y construir algo a partir de prácticamente nada; es un acto creativo propio del género humano. Es liberar la energía personal en el inicio y construcción de una empresa u organización, más que mirar, analizar o describir una. Es tomar riesgos calculados y luego hacer todo lo que sea posible para evitar la falla. Es la habilidad de construir un grupo fundador que complemente las habilidades y talentos del empresario lider. Es la destreza para percibir una oportunidad donde otros ven caos, contradicciones, confusiones y peligros.. Es poseer los conocimientos para identificar, acumular y controlar los recursos (a menudo propiedad de otros) y garantizar que no me falten cuando más los necesito".. (Varela, 1991) A nuestro juicio, esta es una de las definiciones más acertadas y completas de las hasta aqui presentadas.

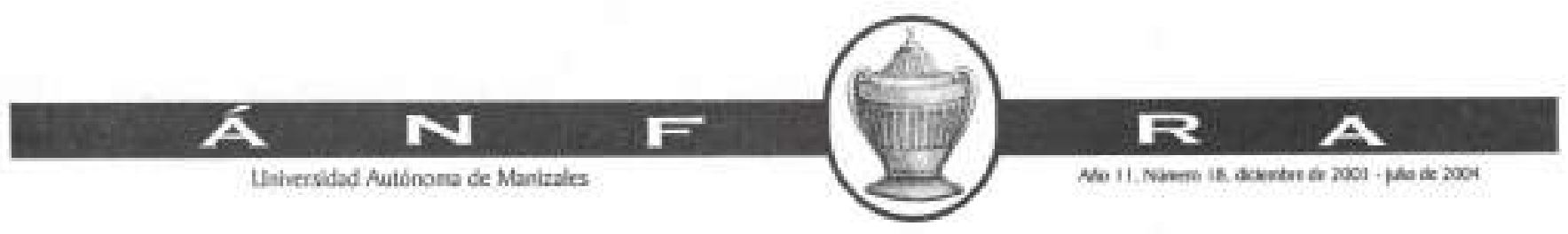


Q. Empresario es quien tiene iniciativa empresarial, entendida como: "aquella cualidad personal que le permite a alguien el iniciar un nuevo negocio o de una manera innovativa y vigorosa, el expandir uno ya existente" (Servicio Nacional de Aprendizaje SENA, 1993). Esta definición es complementaria, sin embargo creemos que no sólo es una cualidad sino que requiere también un conjunto de conocimientos (hasta ahora empiricos) y unas caracteristicas muy propias.

De los escritos de Drucker, Max-Neff, Ohmae y Reichheld extraemos otras ideas, como:

Q. El empresariado es ciencia y arte en continuo cambio porque los conocimientos, la tecnología, los mercados y las estructuras están cambiando muy rápidamente. De aqui la necesidad de planear para la incertidumbre y formar para el riesgo continuo.

\&. El individuo tiene que responsabilizarse más de sí mismo, ser autónomo y libre porque cuando el egresado empieza a trabajar, debe tomar decisiones, nadie lo hace por él.

\& El capital más preciado, hoy, es el conocimiento.

Q. La clave de la productividad de los trabajadores de conocimientos es hacer que se concentren en tareas reales.

2. Poco de objetivo. Se debe ir a lo subjetivo: desarrollar competencias y la empatia, Porque los Economistas Empresariales deben ser sintetizadores que juntan recursos y deben tener la habilidad de "oler" las oportunidades para generar nuevas empresas.

Q. Conocer quién es uno y qué es lo que hace mejor.

\& Los egresados no deben valorarse en función de sus credenciales (títulos) sino en función de su desempeño.

a Los estudiantes deben desarrollar una mente estratégica, más que aprender estrategias; una mente investigadora capaz de resolver problemas; potenciar la creatividad, el análisis y el ingenio.

Q Mejorar las destrezas en 'operaciones intelectuales' y tener un excelente manejo de los 'instrumentos de conocimiento' que les permita adquirir un conocimiento significativo para que no sean sólo repetidores de información mal digerida y hasta obsoleta.

e Capacidad de reconocer patrones para ver to que hay y no lo que uno espera ver.

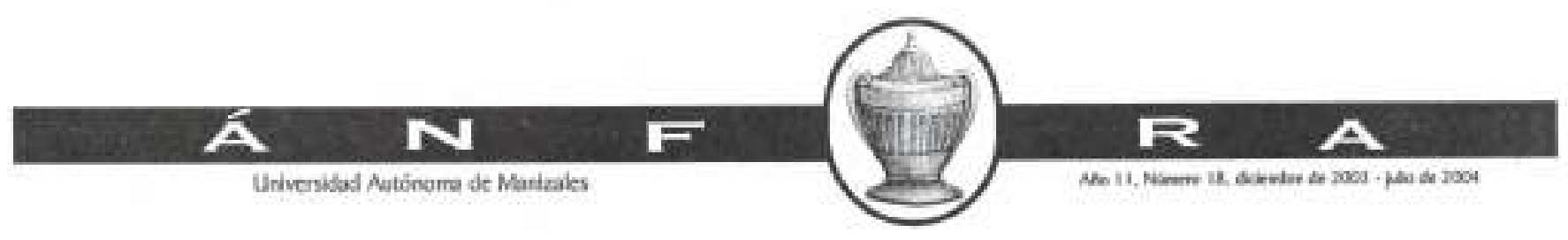


20. El Economista Empresarial debe aprender a hablar el lenguaje corriente $y$ a escuchar a las personas no versadas en empresariado.

Q. Necesidad no sólo de conocer sus propias competencias sino de conocer las capacidades de los hombres y mujeres con quienes comparte sus retos.

飞. El mundo empresarial está pasando de un modelo de mando a un modelo de conocimiento.

Q. El Economista Empresarial debe responsabilizarse de adquirir conocimiento pero sobre todo debe saberlo USAR. Saber ubicar la información y también saber cómo usarla.

e. La formación humanista es indispensable para el economista empresarial y clave del éxito en sus relaciones interpersonales.

Q. Necesidad de aprender a investigar y a pensar en equipo.

\&. Utilidad de cambiar el poder por la responsabilidad, el conocimiento y el liderazgo.

*. La imaginación puede lograr más cosas que el dinero.

de La falta de recursos representa la naturaleza de todo el fuego del desarroilo.

Q. El conocimiento está reemplazando a la autoridad $y$, por tanto, las organizaciones de hoy son planas, no hay jerarquia.

\&. ¿Qué es lo que necesita saber un Economista Empresarial?

Según estas premisas extraídas de los autores mencionados, el perfil del economista empresarial debe apuntar a:

2. Una persona libre, responsable y autónoma. Hábil para tomar decisiones rápidas y seguras. Con alta capacidad para el riesgo y motivación para el logro.

\& Un hombre con mente estratégica, investigador, con capacidad de observar, analizar, ingeniar, describir hechos, explicar, predecir, resolver problemas; curioso, creativo, imaginativo mas no fantasioso, innovador y preparado para poner en juego el conocimiento con el fin de llevar al éxito todas las labores que se le encomienden.

Q2 Un cultivador del pensamiento emotivo y racional, divergente $y$ convergente, crítico y analítico y también del pensamiento práctico, que le

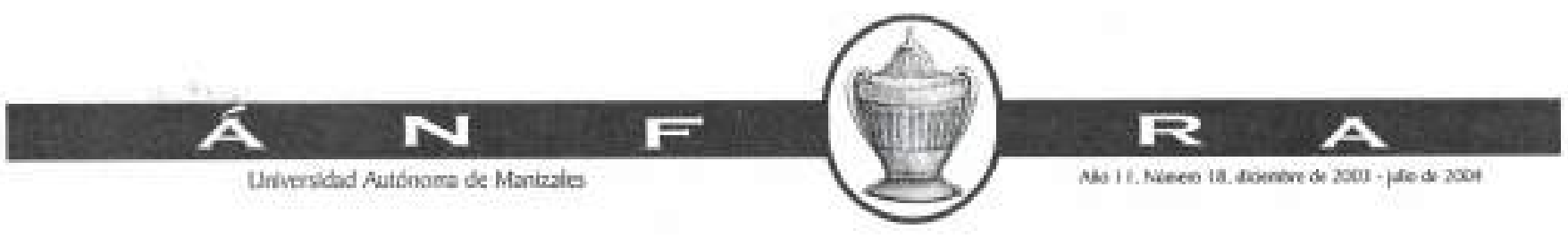


permita interpretar, con criterios propios, los cambios en el entorna social y económico que eventuaimente pudieran afectar el éxito en la creación de empresas.

Q Que aborde la realidad económica y empresarial con una raclonalidad cientifica, pero también con perspicacia, sagacidad y mucha intuición.

2. Identifique, evalúe y priorice los campos en los cuales su conocimiento, habilidades $y$ destrezas puedan generar investigación aplicable al mejoramiento de las empresas y del entorno social en general.

2 Presente planteamientos que permitan la solución a problemas empresariales y económicos regionales y nacionales.

飞. Planifique sistemas empresariales nuevos, determinando los objetivos en función de unos resultados deseados, relacionando su nueva empresa con el sistema de producción regional, nacional e internacional.

Q. Valore los beneficios económicos y sociales que puede reportar la creación de la empresa con el finde orientar sus decisiones.

2. Analice procesos productivos, teniendo en cuenta las exigencias de recursos logisticos, financieros, de talento humano $y$ en general todos aquellos que determina el éxito de la creación de una empresa.

2. Se desempeñe en posiciones de liderazgo en su región, entendiendo por ellas todas aquellas en las cuales su actuación pueda influir en el desenvolvimiento económico y empresarial de su entorno.

2. Genere para si y para la sociedad empleo productivo, a través de la creación o transformación de empresas, que a su vez también represente para la región mejora en el ingreso y bienestar de su gente.

12. Un gran lector $y$ escritor, puesto que al leer $y$ al escribir se adquiere $y$ se propaga el conocimiento.

e $\mathrm{Y}$ sobre todo humano, demasiado humano, para que colabore en la construcción de un mundo mejor para los suyos y para los demás.

Entiéndase, entonces, por empresario, la persona con las capacidades, las habilidades, las destrezas, las intuiciones, las conocimientos y las actitudes propios para la generación de empresas; una mezcla de arte y ciencia, de conocimientos populares y académicos, de doxa y episteme. De esta manera, el empresario que forma el programa de Economia empresarial de la Universidad Autónoma de Manizales tiene una clara diferencia con los jơvenes que se forman en otros programas similares, pues no es una

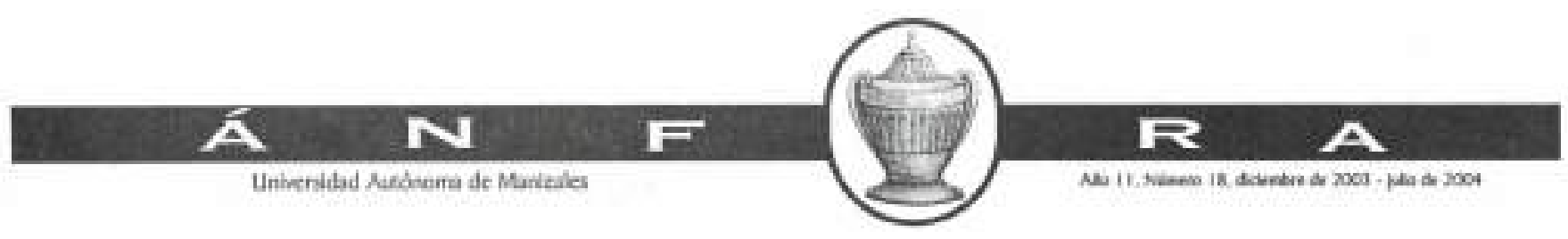


formación para la gerencia, ni con el fin de estudiar el entorno económico solamente, ni para la administración sino para la CREACIÓN Y TRASFORMACIÓN DE EMPRESAS. Sin negar que los estudios complementarios que ofrece el programa hacen viable la posibilidad de dirigir la empresa una vez creada. Estos mismos estudios propician, además, al egresado, las capacidades y habilidades para ser consultor, asesor, intraempresario e investigador.

\section{Propósitos de formación.}

El programa de Economia Empresarial tiene como propósito formar empresarios profesionales, idóneos y competentes para la generación y desarrollo de organizaciones productivas y de serviclos, en un ambiente de incertidumbre; profesionales capaces de contribuir, a través de la aproplación y aplicación de las teorias sobre el empresariado, la economía y la administración, al mejoramiento de la calidad de vida de diversas comunidades.

\section{Conclusianes.}

1. Está claro pues, según lo enunciado hasta el momento, que la Economia empresarial no es un hibrido o una simbiosis entre Economia y Administración, o entre Economia y empresa; por el contrario, es un nuevo campo de conocimiento, o si se quiere, una nueva profesión, con un Objeto de Estudio propio, unos campos de aplicación que van mucho más allíb que los de aquellas disciplinas; un perfil profesional claramente definido y un propósito de formación que no es otro que el de formar EMPRESARIOS.

2. El complemento macrocurricular que la Universidad Autónoma de Manizales ofrece a sus estudiantes permite, en este caso a los alumnos de Economia Empresarial, una formación personal y en ética y valores que consideramos necesaria para el ejercicio de la profesión. El empresario que requiere el pais y la región no puede ser una persona inconsciente de su qué hacer ni de los alcances de sus acciones, ni debe buscar exclusivamente su beneficio personal. De aquí que exista en el programa una constante preocupación por la adquisición de una conciencia social en sus educandos.

3. Este trabajo del equipo de profesores y administrativos de la Facultad de Ciencias Sociales y Empresariales ha sido diseñado con un especial cuidado para responder también con la visión y misión institucionales, pues es

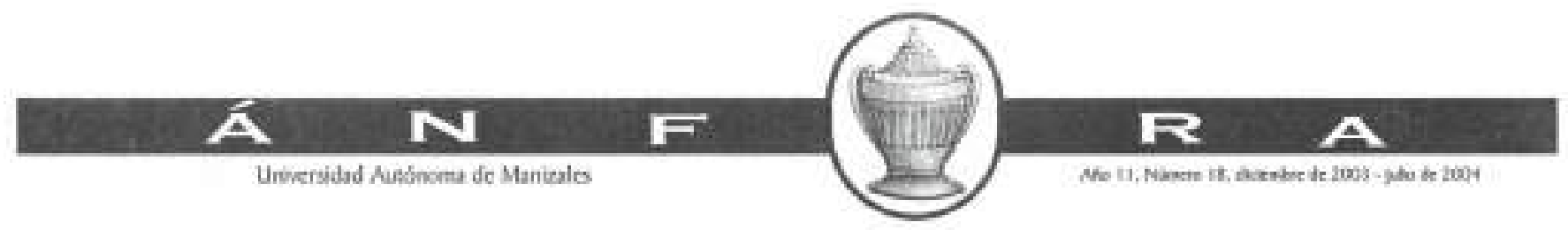


nuestro deber como célula, velar porque el organismo cumpla con los acuerdos generales a los que se han llegado: ser una nueva universidad competitiva con proyección nacional e internacional, comprometida con la excelencia y la innovación permanente; como comunidad educadora, dinamizadora del conocimiento comprometida con la convivencia pacifica y el desarrollo, contribuye a la formación de personas éticas con pensamiento critico e innovador. Es de especial atención para el programa de Economia Empresarial el objetivo especifico de la UAM que reza: "Posicionar la UAM como la universidad de las empresas".

4. La propuesta aqui recogida guarda una perfecta congruencia con las necesidades propias del pais. Pues ante un panorama de desempleo creciente, la solución puede ( $y$ debe) estar en la generación de empresas, es decir, en la conformación de una nueva Clase Empresarial que redimensione y dinamice el modelo actual de desarrollo colombiano. Esa es la pretensión última del programa de Economía Empresarial de la U.,A.M.

\section{BIBLIOGRAFIA}

A.H.A. Archivo Histórico de Antioquia. Documentos Generales, Baidios, Censos y Estadisticas, Acuerdos, Visitas. 1.927.

ANDER-EGG, Ezequiel, Los medios de comunicación al servicio de la educación. Bs.As.: Magisterio del Rio de la Plata. 1992.

ANZOLA, Sérvulo. De la idea a tu empresa, misión de todos. Serie textos universitarios, ICESI. 1998

ARANZADI, Dionisio. El arte de ser empresario hoy. Universidad de Bilbao. Conferencias del programa EXEDU. Desarrollo de la capacidad Empresarial, U de los Andes, 1998

AUSUBEL, D. J. NOVAK y H. HANESIAN. Psicologia educativa: un punto de vista cognoscitivo. México: Trillas, 1988

BARRE, Raymond. El Desarrollo Económico. Fondo de Cultura Económica. Colección Popular. México, 1958

BEjARANo, Jesús Antonio, Modelo de Apertura : Colombia o el Japón Latinoamericano. En : Modelos de Desarrollo Económico-Colombia 1960-82. Editorial La Oveja Negra. Bogotá, mayo de 1982

BOTERO, Libardo y otros. Neoliberalismo y subdesarrollo - un análisis critico de la apertura económica. Ancora editores. Bogotá, 1992

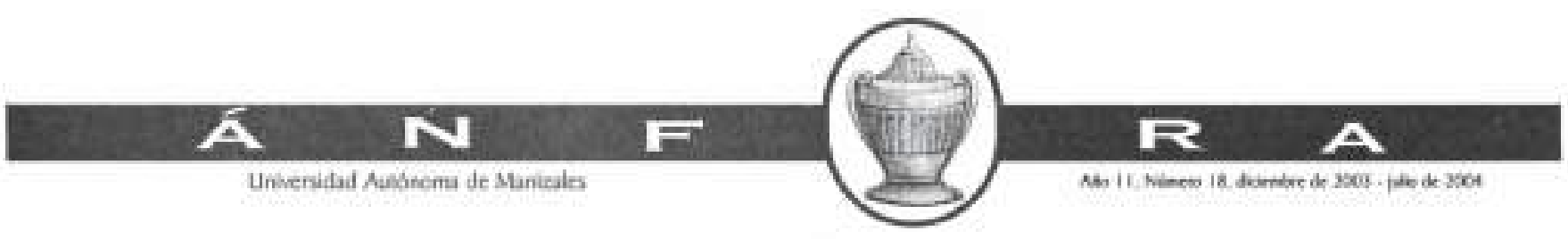


BREW, Roger. El Desarrollo económico de Antioquia desde la independencia hasta 1920. Bogotá: Banco de la República 1977.

BUNGE, Mario. Clencia, técnica y desarrolio. México: Hermes. 1998

CEPAL. Notas sobre la Economia y el Desarrollo. La tarea de los noventa para la región : Fortalecer el desarrollo. Número 590, abrill de 1996

COLCIENCIAS: Bases para un plan del Programa Nacional de Ciencias Sociales y Humanas. 1992.

CORDEIRO, José Lisis, El Desafí Latinoamericano y sus cinco grandes retos. Editorial McGraw Hill. Interamericana de Venezuela S .A. 1995

CUELLAR, Maria Mercedes, "Modernización de la Economia : Modificación del Modelo de Desarrollo*: En ; Revista de Planeación y Desarrollo. Departamento Nacional de Planeación, Volumen XXII, números 3 y 4 . Bogotb, 1990

DAVILA. L. De Guevara, Carios, El Empresariado Colomblano Una Perspectiva Histórica., Pontificia Universidad Javeriana, Facultad de Estudios Interdisciplinarios. Programa de Post-Grado en Economia. 1986.

Pontificia Universidad 3averiana, Facultad de Estudios Interdiciplinarios, Programa de Post-Grado en Economia. 1986.

El Empresariado Colombiano. Una perspectiva histórica, Pontificia Universidad Javeriana Bogotá 1.986.

Historia Empresarial de Colombia, Estudios, Problemas y Perspectivas, Universidad de los Andes, Facultad de Administración 1.991.

DICCIONARIO ENCICLOPÉDICO ECONOMIA Planeta, tomo2. Editorial Planeta, Barcelona 1980

EKELUND, Rober B., HEBERT, Rober F. Historia de la Teoria Económica y de su Método McGraw-Hill México 1.998

FALS BORDA, Oriando. Neoliberalismo y Neoestructuralismo - Núcleo de investigaciones económicas. Bogotá, 1992

FORERO BENAVIDES, Abelardo y otros. Dependencia y Desarrollo. Biblioteca ANIF de Economía. Colombia marzo de 1978

GARAY, Luis Jorge, Liberalismo económico como modelo de Desarrollo, En : Documento Universitas Económica. Volumen 6, Número 3,1989

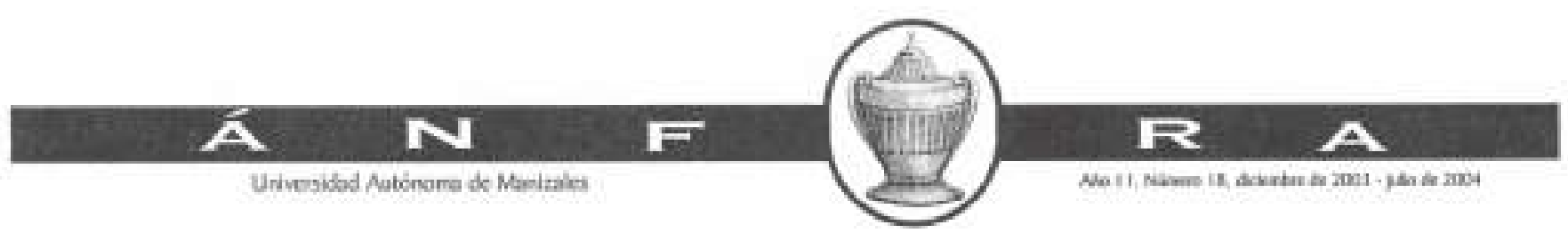


GARCIA, Antonio. Geografía Economía de Colombia. Caldas. Bogotá: Contraloria General de la República, 1937.

GARCIA, Rodrigo. Innovación empresarial. Serie textos universitarios. ICESI.

GILDER, George. El espíritu de la libre empresa. Editorial Lasser Press.

GOLEMAN, Daniel. La inteligencla emocional, Santafé de Bogotá: Panamericana, 1996 .

GOMEZ, SandaHo, La Persona y el Trabajo en la Empresa del Siglo XXI. Biblioteca IESE de Gestión de Empresas. Ediciones Folio, 5.A. Barcelona, 1.997.

JOYANES, Luis. Cibersociedad, Los Retos Sociales ante un Mundo Digital. Editorial MCGraw-HIII/Interamericana de España, S.A.U. Madrid. 1.997.

MADRID, Salvador y RESTREPO, José Manuel. Informe sobre el valle de Aburrá. Archivo Histórico de Antioquía Vol., 343.1888.

MESAROVIC, Mihajlo y PESTEL, Eduard. "La humanidad en la Encrucijada". En : Dependencia y Desarrollo.

MORIN, Edgar. Introducción al pensamiento complejo. Traducción de Marcelo Pakman. Segunda reimpresión. Barcelona: Gedisa, 1998

NORTH, Douglas. Conferencia "Lo primero es colocar la casa en orden", En : Revista ESTRATEGIA, Julio 15 de 1996.

NOVAK, J. Aprendiendo a aprender. Barcelona: Martinez Roca, 1988.

OCAMPO, José Antonio y SARMIENTO PALACIO, Eduardo. ¿ Hada un nuevo modelo de Desarrollo? Un debate. Tercer Mundo Editores - FEDESARROLLO UNLANDES. Bogotá, 1989

ONTORIA, A. Mapas conceptuales: una técnica para aprender. Madrid: Narcea editores. 1994

ORTIZ Sarmiento, Carlos Miguel. La Colonización Antioqueña. Biblioteca de Escritores Caldenses, Manizales Colombia.

PALACIOS, Marco. El café en Colombia. 1850-1970. Bogotå 1979.

PARSONS, James. La Colonización Antioqueña en el occidente de Colombia. Medellin, 1950.

POWDER, Alejandro y otros. Modelos de Desarrolio Económico-Colombia 1960-82. Editorial Oveja Negra. Bogotá, Mayo de 1982

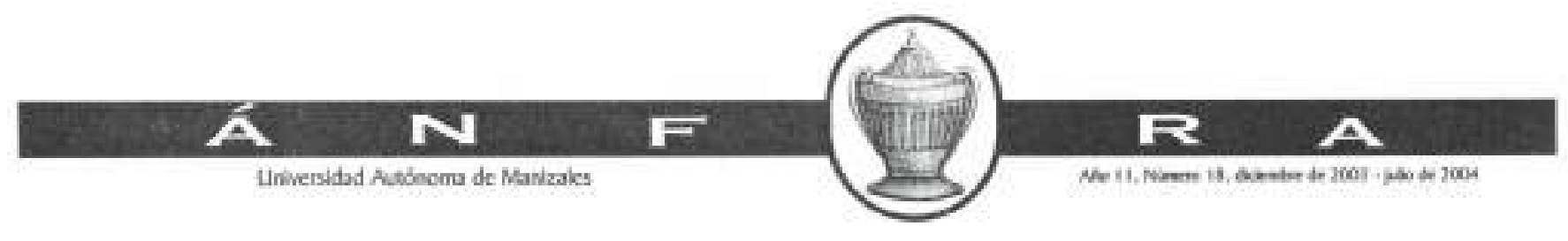


Revista de la CEPAL No 56, agosto 1995, p. 13-24

RESTREPO, A., Ignacio, 50 Años Del Desarrollo Económico de Manizales. Talleres de Editores S.A. 1995.

RICARD, Joan Enric. "Nuevas Formas Organizativas: Funcionamiento" Publicado en Cómo Prepararse para las Organizaciones del Futuro. Biblioteca IESE de Gestión Empresarial. Universidad de Navarra. Ediciones Folio, Barcelona, 1.997.

RODRIGUEZ, Becerra Manuel., El Empresario Industrial del Viejo Caldas, Facultad de Administración Universidad de los Andes. 1993.

RODRIGUEZ, B. Manuel El Empresario Industrial del Viejo Caldas. Facultad de Administración Universidad de los Andes. Bogotă 1.993

ROLL, Eric. Historia de las Doctrinas Económicas, Editorial. Fondo de Cultura Económica. Colombia, 1993

SANCHEZ RUNDE, Carlos J. Dirección Estratégica de Recursos Humanos. Biblioteca IESE de Gestión de Empresas. Ediciones Folio, S.A. Barcelona, 1.997 .

SARMIENTO PALACIO, Eduardo. Los nuevos desafios del desarrollo Fundamentos y Politicas. Tercer Mundo editores, Ediciones UNIANDES. Bogotá, Marzo de 1990

SPENCER, M.H., Economia Contemporánea Edi Reverte S.A.Barcelona 1,984

TEZANOS de Araceli: Colección pedagogia S.XXI. Aproximaciones Metodológicas para la enseñanza del enfoque cualitativo interpretativo para la investigación social. Ediciones Antropos, primera edición 1998.

UNIVERSIDAD NACIONAL DE COLOMBIA: La Investigación Acción Participativa Inicios y Desarrollos, Cooperativa editorial MAGISTERIO. 1997.

VYGOTSKI, L,S. Desarrollo de los procesos psicológicos superiores. Barcelona: Crítica. 1979.

Pensamiento y lenguaje. Teoria del desarrollo cultural de las funciones psiquicas. Bs As: La pléyade. 1985

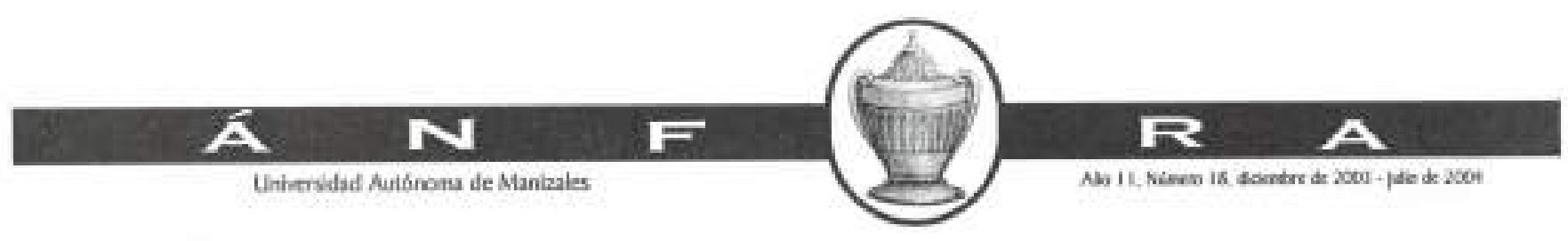

